\title{
A Hamiltonian approach to the cohomogeneity one Ricci soliton equations and explicit examples of non-Kähler solitons
}

Alejandro Betancourt de la Parra, Andrew S. Dancer, and McKenzie Y. Wang

Citation: Journal of Mathematical Physics 57, 122501 (2016);

View online: https://doi.org/10.1063/1.4972216

View Table of Contents: http://aip.scitation.org/toc/jmp/57/12

Published by the American Institute of Physics

\section{Articles you may be interested in}

Exact solutions of Friedmann equation

Journal of Mathematical Physics 57, 122503 (2016); 10.1063/1.4972836

KP flows and quantization

Journal of Mathematical Physics 57, 123503 (2016); 10.1063/1.4972024

Fractional Schrödinger equation with Riesz-Feller derivative for delta potentials Journal of Mathematical Physics 57, 123506 (2016); 10.1063/1.4972291

Geometric quantization of Dirac manifolds

Journal of Mathematical Physics 57, 123507 (2016); 10.1063/1.4972779

Projective loop quantum gravity. I. State space

Journal of Mathematical Physics 57, 122304 (2016); 10.1063/1.4968205

Reductions in finite-dimensional integrable systems and special points of classical r-matrices Journal of Mathematical Physics 57, 123504 (2016); 10.1063/1.4972021

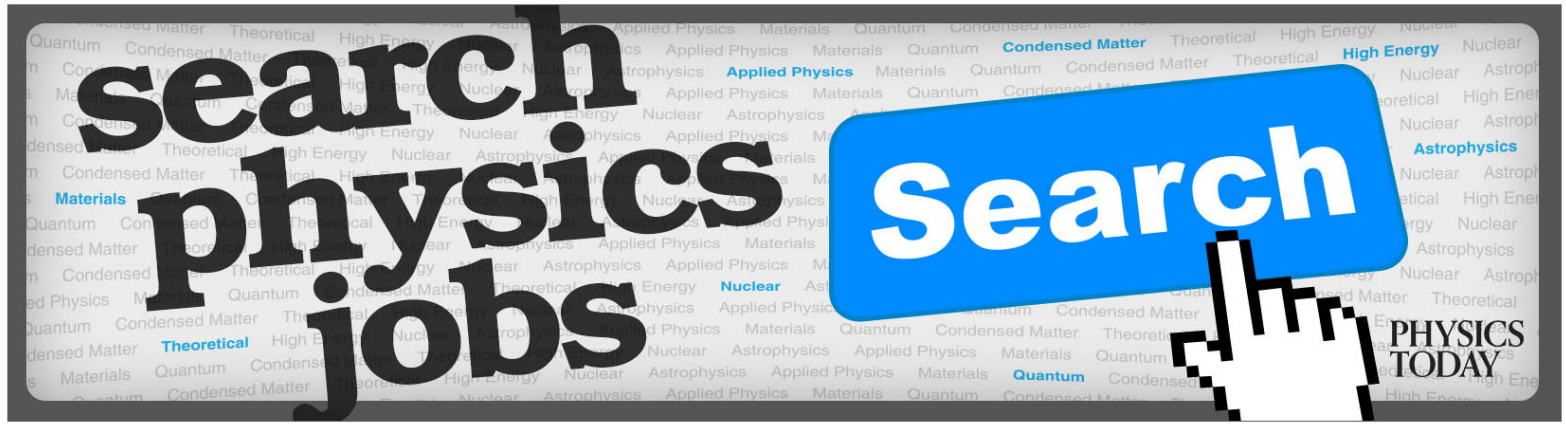




\title{
A Hamiltonian approach to the cohomogeneity one Ricci soliton equations and explicit examples of non-Kähler solitons
}

\author{
Alejandro Betancourt de la Parra, ${ }^{1, a)}$ Andrew S. Dancer, ${ }^{2, b)}$ \\ and McKenzie Y. Wang ${ }^{3, c)}$ \\ ${ }^{1}$ Mathematical Institute, Andrew Wiles Building, Oxford University, \\ OX2 6GG, United Kingdom \\ ${ }^{2}$ Jesus College, Oxford University, OX1 3DW, United Kingdom \\ ${ }^{3}$ Department of Mathematics and Statistics, McMaster University, Hamilton, \\ Ontario, L8S 4K1, Canada
}

(Received 6 October 2015; accepted 1 December 2016; published online 20 December 2016)

\begin{abstract}
We show how to view the equations for a cohomogeneity one Ricci soliton as a Hamiltonian system with a constraint. We investigate conserved quantities and superpotentials and use this to find some explicit formulae for Ricci solitons not of Kähler type in five dimensions. Published by AIP Publishing. [http://dx.doi.org/10.1063/1.4972216]
\end{abstract}

\section{INTRODUCTION}

Einstein metrics are the critical points of the Einstein-Hilbert action restricted to the set of Riemannian metrics with fixed volume. In the context of metrics of cohomogeneity one, this variational characterization and the invariance under diffeomorphisms give rise to an interesting structure for the Einstein equations-they can be written as a Hamiltonian system with a constraint (the vanishing of the Hamiltonian). This structure has proved useful in identifying conserved quantities for certain cases of the Einstein system and for finding superpotentials which define first order subsystems of the Einstein equations (see Refs. 7-9, for example). Frequently, these quantities in turn lead to explicit solutions of the Einstein equations, and the associated first order subsystems single out solutions with special holonomy.

In an analogous manner, Perelman's $\mathcal{F}$ and $\mathcal{W}$ functionals ${ }^{17}$ possess diffeomorphism and scale invariance properties, and consideration of their first variations which preserve the dilaton measure leads to the gradient Ricci soliton (GRS) equations. In this paper we will investigate the cohomogeneity one case using the framework of Ref. 12 and put the gradient Ricci soliton equations in Hamiltonian form with a constraint. We then focus on the case of steady solitons and consider the situation in which the principal orbit has multiplicity free isotropy representation.

Let us denote the Hamiltonian (to be constructed in Sec. III) by $\mathcal{H}$. Recall that a generalised first integral is a function on momentum phase space whose Poisson bracket with the Hamiltonian lies in the ideal generated by $\mathcal{H}$. As well, a superpotential is a $C^{2}$ function $f$ on configuration space that gives a time-independent solution of the Hamilton-Jacobi equation, i.e., $\mathcal{H}\left(q, d f_{q}\right)=0$. In the situation of the Bryant solitons, we find both generalised first integrals and superpotentials when the dimension is either 2 or 5. This leads to new explicit formulae for the Bryant soliton in dimension 5. We also find superpotentials for the gradient Ricci soliton equations on double warped products $\mathbb{R}^{3} \times \Sigma$ where $\Sigma=S^{2}$ or $\mathbb{R P}^{2}$ and for certain complex line bundles over a product of Fano Kähler-Einstein manifolds. The associated first order systems in the latter case correspond to the

\footnotetext{
a) Present address: Centro de Investigación en Matemáticas (CIMAT), 36000 Guanajuato Gto., Mexico. E-mail address: betancourtde@maths.ox.ac.uk

b) E-mail address: dancer@maths.ox.ac.uk

c) E-mail address: wang@mcmaster.ca
} 
Kähler condition, and explicit complete steady Kähler Ricci solitons were obtained in Ref. 12 (Theorem 4.20). Here we use the superpotentials in the former case to obtain explicit complete steady gradient Ricci soliton structures on $\mathbb{R}^{3} \times \Sigma$. The existence of these non-Kählerian solitons was obtained previously using dynamical systems methods (cf. Refs. 16 and 10), but explicit complete solutions had not, as far as we are aware, been found previously.

To our knowledge, the five-dimensional examples mentioned above are the first explicit examples of inhomogeneous non-Kähler, non-Einstein Ricci solitons not of Gaussian type.

Finally, we mention that in Sec. V we prove a non-existence theorem (see Proposition 13) for superpotentials of exponential type assuming a certain natural condition. We refer the reader to Sec. V for the description of this condition. It is noteworthy, however, that superpotentials satisfying this condition do exist in the Ricci-flat case and most of the time when they occur, the associated first order systems are equivalent to the condition that the metrics have special holonomy. So this non-existence result may be interpreted as an indication of the greater rigidity of the soliton equation.

\section{BASIC FACTS}

Let $(M, \bar{g})$ denote a connected Riemannian manifold of dimension $n+1$ on which a compact Lie group $G$ acts via isometries with cohomogeneity one, i.e., with one-dimensional orbit space. We will assume that the orbit space is an interval $I \subset \mathbb{R}$ and that the principal orbit type is given by $P:=G / K$, where $K$ is a closed subgroup of $G$. We further assume that there is at least one special orbit, which, without loss of generality, is of the form $G / H$ where $K \subset H$ and $H$ is closed in $G$. The cohomogeneity one condition then implies that $H / K$ is diffeomorphic to a sphere $S^{k}$. By choosing a constant speed geodesic that intersects one (hence all) principal orbits orthogonally, we obtain a diffeomorphism of the open dense submanifold $M_{0} \subset M$ consisting of all the principal orbits with int $I \times P$. Then the metric $\bar{g}$ takes the form

$$
\bar{g}=d t^{2}+g_{t},
$$

where $t$ is the geodesic parameter and $g_{t}$ is a one-parameter family of $G$-invariant metrics on $G / K$.

Let $L_{t}$ denote the shape operator of the hypersurface $\{t\} \times P$, regarded as a $g_{t}$-symmetric endomorphism of $T(G / K)$. Also, let $r_{t}$ denote the Ricci endomorphism defined by $\operatorname{Ric}\left(g_{t}\right)(X, Y)=$ $\left.g_{t}\left(r_{t}(X), Y\right)\right)$, where $X, Y$ are tangent to $G / K$. Then $\dot{g}_{t}=2 g_{t} \circ L_{t}$, where each $g_{t}$ is regarded as an endomorphism of $T(G / K)$ via the choice of a fixed $G$-invariant metric $Q$ on $G / K$. The Levi-Civita connections of $\bar{g}$ (respectively, $g_{t}$ ) will be denoted by $\bar{\nabla}$ (respectively, $\nabla$ ). The relative volume $v$ is defined by $d \mu_{g_{t}}=v(t) d \mu_{Q}$.

The static equation for a gradient Ricci soliton $(\mathrm{GRS})(M, \bar{g}, u)$ is

$$
\operatorname{Ric}(\bar{g})+\operatorname{Hess}_{\bar{g}} u+\frac{\epsilon}{2} \bar{g}=0 .
$$

$\bar{g}$ is called the soliton metric and $u: M \rightarrow \mathbb{R}$ is a smooth function called the soliton potential. We shall say that a gradient Ricci soliton is trivial if the soliton metric is Einstein. The example of the Gaussian soliton shows that the potential could nevertheless be non-trivial.

In the cohomogeneity one situation, Eq. (2.2) becomes, on $M_{0} \approx$ int $I \times P$, the system

$$
\begin{aligned}
r_{t}-\dot{L}-(\operatorname{tr} L-\dot{u}) L+\frac{\epsilon}{2} \mathbb{I} & =0, \\
-\operatorname{tr}\left(L^{2}\right)-\operatorname{tr}(\dot{L})+\ddot{u}+\frac{\epsilon}{2} & =0, \\
d(\operatorname{tr} L)+\delta^{\nabla} L & =0,
\end{aligned}
$$

where the soliton potential $u$ is regarded both as a function on $M$ and as a function of $t$, and $\delta^{\nabla}$ is the codifferential for $T^{*}(G / K)$-valued 1-forms.

Note that Eq. (2.3) above represents the components of the gradient Ricci soliton equation tangent to $G / K$, Eq. (2.4) is the equation in the $\partial / \partial t$ direction, and Eq. (2.5) represents the mixed directions. In fact, by $G$-invariance, $\operatorname{tr} L$ is always constant in the $G / K$ directions, but we have 
included it in (2.5) because the above system also holds under suitable assumptions when $M$ is constructed out of an equidistant family of hypersurfaces with possibly no symmetries (cf. Ref. 11, Remark 2.18). This possibility, incidentally, is a frequently misunderstood aspect of the work of the last two authors.

For the general GRS equation, there is a fundamental conservation law which was observed by Hamilton ${ }^{15}$ and Ivey. ${ }^{16}$ In the cohomogeneity one setting, this conservation law becomes

$$
\ddot{u}+(-\dot{u}+\operatorname{tr} L) \dot{u}-\epsilon u=C,
$$

where $C$ is a fixed constant. Using (2.4) and the trace of (2.3), we can rewrite (2.6) in the form

$$
S+\operatorname{tr}\left(L^{2}\right)-(-\dot{u}+\operatorname{tr} L)^{2}+(n-1) \frac{\epsilon}{2}=C+\epsilon u,
$$

where $S$ is the scalar curvature of the principal orbits and $C$ is the same constant. Recall that in Ref. 6 we introduced the quantities

$$
\xi:=-\dot{u}+\operatorname{tr} L, \quad \mathcal{E}=C+\epsilon u
$$

and rewrote the conservation law in the form

$$
\ddot{\mathcal{E}}+\xi \dot{\mathcal{E}}-\epsilon \mathcal{E}=0 .
$$

Note that $\xi$ is a natural quantity to consider, as it is just the mean curvature of the dilaton volume element $e^{-u} d \mu_{\bar{g}}$. Furthermore, for functions of the variable $t$, the operator $\ddot{f}+\xi \dot{f}$ is just the $u$-Laplacian in the theory of metric measure spaces.

Finally we recall that the scalar curvature of the metric $\bar{g}$ is given by

$$
\bar{R}=-2 \operatorname{tr}(\dot{L})-\operatorname{tr}\left(L^{2}\right)-(\operatorname{tr} L)^{2}+S .
$$

Using the trace of (2.3) followed by (2.7) we obtain

$$
\begin{aligned}
\bar{R} & =-S+(\operatorname{tr} L)^{2}-\operatorname{tr}\left(L^{2}\right)-2 \dot{u} \operatorname{tr} L-\epsilon n \\
& =-C-\epsilon u-\dot{u}^{2}-\frac{\epsilon}{2}(n+1),
\end{aligned}
$$

which is just the cohomogeneity one case of Hamilton's identity (cf. Ref. 15, p. 84).

\section{A HAMILTONIAN FORMULATION OF THE COHOMOGENEITY ONE GRS EQUATIONS}

In this section we will construct a Hamiltonian on an appropriate symplectic manifold such that integral curves of the associated Hamiltonian vector field lying on the zero energy hypersurface correspond to solutions of the cohomogeneity one gradient Ricci soliton equations modulo smoothness considerations. The Einstein case was discussed in Ref. 7 and the present case is essentially analogous.

Given a principal orbit $G / K$, we first fix an $\mathrm{Ad}_{K}$-invariant decomposition

$$
\mathfrak{g}=\mathfrak{f} \oplus \mathfrak{p}
$$

of the Lie algebra $\mathfrak{g}$ of $G$, so that $\mathfrak{p} \approx T_{[K]}(G / K)$. Recall that in Sec. II we have fixed a background invariant metric $Q$ on $\mathfrak{p}$. Now let $\mathcal{C}$ be the configuration space $S_{+}^{2}(\mathfrak{p})^{K} \times \mathbb{R}$ where $S_{+}^{2}(\mathfrak{p})^{K}$ denotes the space of all $\operatorname{Ad}(K)$-invariant, positive-definite, symmetric endomorphisms of $\mathfrak{p}$ with respect to $Q$. Via the relation $g_{t}(X, Y)=Q\left(q_{t}(X), Y\right)$, a path $\left(q_{t}, u(t)\right) \in \mathcal{C}$ corresponds to a one-parameter family of $G$-invariant metrics on $G / K$ together with a soliton potential function.

The velocity phase space is $T \mathcal{C}=\left(S_{+}^{2}(\mathfrak{p})^{K} \times \mathbb{R}\right) \times\left(S^{2}(\mathfrak{p})^{K} \times \mathbb{R}\right)$ and we will denote a typical element in it by $(q, u, \dot{q}, \dot{u})$. In order to write down a suitable Lagrangian function, we introduce the following non-degenerate symmetric bilinear form on $\operatorname{End}(\mathfrak{p}) \times \mathbb{R}$ :

$$
\left\langle\left(h_{1}, \eta_{1}\right),\left(h_{2}, \eta_{2}\right)\right\rangle:=\frac{1}{2}\left(\operatorname{tr}\left(h_{1}\right) \operatorname{tr}\left(h_{2}\right)-\operatorname{tr}\left(h_{1} h_{2}\right)\right)+2 \eta_{1} \eta_{2}-\left(\operatorname{tr}\left(h_{1}\right) \eta_{2}+\operatorname{tr}\left(h_{2}\right) \eta_{1}\right) .
$$

The induced symmetric bilinear form on $\operatorname{End}(\mathfrak{p})^{*} \times \mathbb{R}^{*}$ will be denoted by $\langle\cdot, \cdot\rangle^{*}$. These forms are, up to a minus sign, extensions of the symmetric bilinear forms on $\operatorname{End}(\mathfrak{p})$ and $\operatorname{End}(\mathfrak{p})^{*}$ introduced 
in Ref. 7 (see Eq. (1.15) there). As is easily checked, the extended forms also have Lorentz signature $(-, \ldots,-,+)$.

Recall also that $\mathrm{Gl}(\mathfrak{p})$ acts on the left of $\operatorname{End}(\mathfrak{p})$ (by composition), and we can extend this action to $\operatorname{End}(\mathfrak{p}) \times \mathbb{R}$ by making $\operatorname{Gl}(\mathfrak{p})$ act trivially on $\mathbb{R}$. In particular, for $(p, \phi) \in S^{2}\left(\mathfrak{p}^{*}\right)^{K} \times \mathbb{R}^{*}$, $q \in S_{+}^{2}(\mathfrak{p})^{K}$, and $(h, \eta) \in S^{2}(\mathfrak{p})^{K} \times \mathbb{R}$, we have

$$
\left(q^{-1} \cdot(p, \phi)\right)(h, \eta)=p(q \cdot h)+\phi(\eta) .
$$

We now introduce the Lagrangian

$\mathcal{L}(q, u, \dot{q}, \dot{u})=e^{-u} v(q)\left(\tau\left(\frac{1}{2}\left\langle q^{-1} \dot{q}, q^{-1} \dot{q}\right\rangle+\dot{u}^{2}-\dot{u} \operatorname{tr}\left(q^{-1} \dot{q}\right)+S(q)\right)+\lambda(u-n-1)+E\right)$,

where $v(q)$ is the relative volume and $S(q)$ the scalar curvature associated to the metric $q$ on $G / K$. The parameter $E$ is a Lagrange multiplier associated to the constant energy condition that will be presently introduced, and the parameters $\tau$ and $\lambda$ will eventually be set equal to 1 and $-\epsilon$, respectively.

Remark 1. The above Lagrangian can be derived from Perelman's $\mathcal{W}$-functional ${ }^{17}$

$$
\mathcal{W}(\bar{g}, u, \tau)=\frac{1}{(4 \pi \tau)^{N / 2}} \int_{M}\left(\tau\left(\bar{R}+|\nabla u|^{2}\right)-\epsilon(u-N)\right) e^{-u} d \mu_{\bar{g}},
$$

in which $\bar{R}$ is the scalar curvature of $\bar{g}$ and $N=\operatorname{dim} M$. Strictly speaking, Perelman considered only the $\epsilon=-1$ case for compact $M$. The above modification was introduced, for example, in Ref. 5, see p. 229.

In the situation where $M$ is a cohomogeneity one manifold, we write its dimension $N$ as $n+1$ and substitute Eq. (2.10) into the above integral. Recall that $\operatorname{tr} L$ is the logarithmic derivative of the relative volume $v$. Then, integrating by parts formally to get rid of second derivative terms, one obtains (3.3). The usual constraint

$$
\frac{1}{(4 \pi \tau)^{N / 2}} \int_{M} e^{-u} d \mu_{\bar{g}}=\text { const }
$$

is accounted for by the introduction of the multiplier $E$ above. In order to treat the cases of steady and expanding solitons simultaneously with the shrinking case, we have further introduced the parameter $\lambda=-\epsilon$ and have suppressed the multiplicative factor $\operatorname{vol}(Q) /(4 \pi \tau)^{n+1}$ in $\mathcal{W}$. (Note that $d \mu_{\bar{g}}=v(q) \operatorname{vol}(Q) d t$.) An important advantage of using the alternative Lagrangian (3.3) as a starting point in the Hamiltonian approach is that we can take $M$ to be non-compact or even incomplete.

The momentum phase space is the cotangent bundle $T^{*} \mathcal{C}=\left(S_{+}^{2}(\mathfrak{p})^{K} \times \mathbb{R}\right) \times\left(S^{2}\left(\mathfrak{p}^{*}\right)^{K} \times \mathbb{R}^{*}\right)$ equipped with the canonical symplectic structure. A typical element of $T^{*} \mathrm{C}$ will be denoted by $(q, u, p, \phi)$. The Legendre transformation is defined by the equations

$$
\begin{aligned}
& p(h)=\mathcal{L}_{\dot{q}}(h)=e^{-u} v(q) \tau\left(\left\langle q^{-1} \dot{q}, q^{-1} h\right\rangle-\dot{u} \operatorname{tr}\left(q^{-1} h\right)\right), \\
& \phi(\eta)=\mathcal{L}_{\dot{u}}(\eta)=e^{-u} v(q) \tau\left(2 \dot{u} \eta-\operatorname{tr}\left(q^{-1} \dot{q}\right) \eta\right) .
\end{aligned}
$$

The associated Hamiltonian is then given by

$$
\mathcal{H}=p(\dot{q})+\phi(\dot{u})-\mathcal{L} .
$$

More explicitly, $\mathcal{H}$ can be written as

$$
\mathcal{H}=\frac{e^{u}}{v(q)} \frac{\tau}{2}\left\langle q^{-1} \cdot(p, \phi), q^{-1} \cdot(p, \phi)\right\rangle^{*}+\frac{v(q)}{e^{u}}(-E+\lambda(n+1-u)-\tau S(q)),
$$

where we have used the fact that the covector $q^{-1} \cdot(p, \phi)$ is dual to $e^{-u} v(q)\left(q^{-1} \dot{q}, \dot{u}\right)$ with respect to the symmetric bilinear form (3.1). The above Hamiltonian should be compared to (1.9) in Ref. 7. Equivalently, via the inverse Legendre transformation, we have

$$
\mathcal{H}=v(q) e^{-u}\left(\tau\left(2\langle L, L\rangle+\dot{u}^{2}-2 \dot{u} \operatorname{tr} L\right)-E+\lambda(n+1-u)-\tau S(q)\right) .
$$


Since (3.1) specializes to $2\langle L, L\rangle=(\operatorname{tr} L)^{2}-\operatorname{tr}\left(L^{2}\right)$, the zero energy condition can be rewritten as

$$
\operatorname{tr}\left(L^{2}\right)-(-\dot{u}+\operatorname{tr} L)^{2}+S+\frac{\lambda}{\tau} u=\frac{1}{\tau}(-E+\lambda(n+1)),
$$

which is just the conservation law (2.7) if we set $\tau=1$ and $\lambda=-\epsilon$. The constant $C$ in (2.7) is then given by $-\left(E+\frac{\epsilon}{2}(n+3)\right)$.

Remark 2. In the case of a gradient Ricci soliton of cohomogeneity one, if we use Eq. (2.12) together with the above relation between $E$ and $C$ in the $\mathcal{W}$-functional, we obtain

$$
\mathcal{W}(\bar{g}, u, 1)=\frac{1}{(4 \pi)^{(n+1) / 2}} \int_{M}(E+\epsilon(n+2)-2 \epsilon u) e^{-u} d \mu_{\bar{g}} .
$$

Let us choose the normalization $(4 \pi)^{-(n+1) / 2} \int_{M} e^{-u} d \mu_{\bar{g}}=1$. For the steady case (i.e., $\epsilon=0$ ) one can then interpret $E$ as Perelman's energy $\mathcal{F}(\bar{g}, u)$. For the shrinking case $(\epsilon<0)$, we obtain

$$
\mathcal{W}(\bar{g}, u, 1)=\left(E+\frac{\epsilon}{2}(n+2)\right)-\frac{2 \epsilon}{(4 \pi)^{(n+1) / 2}} \int_{M} u e^{-u} d \mu_{\bar{g}},
$$

where the last integral is the classical entropy.

As in Ref. 7, the geometric significance of the zero energy condition is given by the following.

Proposition 3. Assume that there is a singular orbit $G / H$ with dimension strictly smaller than that of the principal orbits. An integral curve of the Hamiltonian vector field that corresponds (under the Legendre transformation) to a $C^{2}$ Riemannian metric $\bar{g}=d t^{2}+g_{t}$ and a potential function $u(t)$ defined in an open neighbourhood of the singular orbit must actually lie on the variety $\{\mathcal{H}=0\}$.

Proof. We may assume that the singular orbit is placed at $t=0$. The Hamiltonian is constant along any integral curve. To evaluate the value of the constant, note that the smoothness conditions imply that $v(0)=0, \dot{u}(0)=0$, and $u(0)$ is finite. By the proof of Lemma 1.10 in Ref. 7, as $t$ tends to 0 , all terms on the right of (3.7) tend to 0 except possibly the term involving $v \dot{u} \operatorname{tr} L$. But $\dot{u} \operatorname{tr} L$ tends to a finite constant, so in fact the remaining term also tends to 0 .

Regarding the zero set $Z_{\mathcal{H}}:=\{\mathcal{H}=0\}$, we have the following.

Proposition 4. When $\lambda \neq 0$ the variety $z_{\mathcal{H}}$ is a smooth hypersurface in momentum phase space. It is also smooth when $\lambda=0$ and the principal orbit $G / K$ is not a torus; otherwise the possible singular points are of the form $(q, u, p, \phi)=(q, u, 0,0)$ where $q$ corresponds to a $G$-invariant flat metric on $G / K$.

Proof. We need to examine the differential $d \mathcal{H}$ at points $(q, u, p, \phi)$ in $\mathcal{Z}_{\mathcal{H}}$. The partial derivative

$$
\mathcal{H}_{(p, \phi)}(\alpha, \beta)=\frac{e^{u}}{v} \tau\left\langle q^{-1} \cdot(p, \phi), q^{-1} \cdot(\alpha, \beta)\right\rangle^{*} .
$$

Since $\langle$,$\rangle is non-degenerate, the above partial derivative vanishes (for all (\alpha, \beta)$ ) if and only if $(p, \phi)=(0,0)$. The vanishing of $\mathcal{H}$ now implies that $E=-(\tau S+\lambda(u-n-1))$. Using these two facts in the partial derivative of $\mathcal{H}$ with respect to $u$ we obtain

$$
\mathcal{H}_{u}(\eta)=-\lambda \eta v e^{-u}
$$

which vanishes (for all $\eta$ ) only if $\lambda=0$. This gives the first statement of the proposition.

If $\lambda=0$, using $(p, \phi)=(0,0)$, we obtain

$$
\mathcal{H}_{q}(h)=e^{-u} d v_{q}(h)(-E+\lambda(n+1-u)-\tau S)-e^{-u} v\left(\tau(d S)_{q}(h)\right) .
$$

Since the first term vanishes by the zero energy condition, the vanishing of $\mathcal{H}_{q}$ reduces to the vanishing of $(d S)_{q}$. As in the proof of Proposition 1.15 in Ref. 7, we conclude that $q$ is a Ricci-flat $G$-invariant metric on the principal orbit. It is well-known that the principal orbit must then be a torus. 
Since the solution curves of the Euler-Lagrange equation for $\mathcal{L}$ correspond to the integral curves of the canonical equations for $\mathcal{H}$, we proceed to determine explicitly the components of the Euler-Lagrange equation and show that they yield (2.3) and (2.4) if one further assumes the zero energy condition.

We begin with

$$
\mathcal{L}_{(\dot{q}, \dot{u})}(h, \eta)=\tau v e^{-u}\left(2 \dot{u} \eta-2(\operatorname{tr} L) \eta+2\left\langle L, q^{-1} h\right\rangle-\dot{u} \operatorname{tr}\left(q^{-1} h\right)\right) .
$$

It follows that

$$
\begin{aligned}
\frac{d}{d t} \mathcal{L}_{(\dot{q}, \dot{u})}(h, \eta)= & \tau v e^{-u}\left[(-\dot{u}+\operatorname{tr} L)\left(2 \eta(\dot{u}-\operatorname{tr} L)+2\left\langle L, q^{-1} h\right\rangle-\dot{u} \operatorname{tr}\left(q^{-1} h\right)\right)\right. \\
& \left.+2 \eta(\ddot{u}-\operatorname{tr}(\dot{L}))+2\left\langle\dot{L}, q^{-1} h\right\rangle-2\left\langle L, q^{-1} \dot{q} q^{-1} h\right\rangle-\ddot{u} \operatorname{tr}\left(q^{-1} h\right)+\dot{u} \operatorname{tr}\left(q^{-1} \dot{q} q^{-1} h\right)\right] .
\end{aligned}
$$

We also have

$$
\begin{aligned}
\mathcal{L}_{(q, u)}(h, \eta)= & v e^{-u}\left(\frac{1}{n-1}\left\langle I, q^{-1} h\right\rangle-\eta\right)\left[\tau\left(2\langle L, L\rangle+\dot{u}^{2}-2 \dot{u} \operatorname{tr} L+S\right)+\lambda(u-n-1)+E\right] \\
& +v e^{-u}\left[\lambda \eta+\tau\left(2\left\langle q^{-1} h, r_{q}\right\rangle-\operatorname{tr}\left(q^{-1} h\right) S+2 \dot{u} \operatorname{tr}\left(q^{-1} h L\right)-4\left\langle L, q^{-1} h L\right\rangle\right)\right],
\end{aligned}
$$

where we have used Lemma 1.12 in Ref. 7 and $r_{q}$ denotes the Ricci endomorphism of the metric $q$.

Now the Euler-Lagrange equation

$$
\frac{d}{d t} \mathcal{L}_{(\dot{q}, \dot{u})}(h, \eta)=\mathcal{L}_{(q, u)}(h, \eta)
$$

must hold for all $h \in S^{2}(\mathfrak{p})^{K}$ and all $\eta \in \mathbb{R}$. Setting $h=0$ and simplifying, we obtain the equation

$$
2 \ddot{u}-2 \operatorname{tr}(\dot{L})-(-\dot{u}+\operatorname{tr} L)^{2}-\operatorname{tr}\left(L^{2}\right)+S+\frac{\lambda}{\tau} u=\frac{-E+\lambda(n+2)}{\tau} .
$$

If we then apply the zero energy condition (3.8), we obtain

$$
\ddot{u}-\operatorname{tr}(\dot{L})-\operatorname{tr}\left(L^{2}\right)=\frac{\lambda}{2 \tau},
$$

which becomes Eq. (2.4) if we set $\tau=1$ and $\lambda=-\epsilon$.

If instead we set $\eta=0$ in the Euler-Lagrange equation, then after some amount of simplification we obtain the equation

$$
\dot{L}+(\operatorname{tr} L) L-\dot{u} L-r=\left(2\langle L, L\rangle+2 \ddot{u}-\dot{u}^{2}-S+\frac{\lambda(u-n-1)+E}{\tau}\right) \frac{I}{2(n-1)} .
$$

Taking the trace of (3.11) yields

$$
\operatorname{tr}(\dot{L})+(\operatorname{tr} L)^{2}-\dot{u}(\operatorname{tr} L)-S=\frac{n}{2(n-1)}\left((\operatorname{tr} L)^{2}-\operatorname{tr}\left(L^{2}\right)+2 \ddot{u}-\dot{u}^{2}-S+\frac{\lambda(u-n-1)+E}{\tau}\right) .
$$

If we substitute (3.10) and the zero energy condition (3.8) into this equation, then after some simplification we deduce

$$
2 \ddot{u}-\dot{u}^{2}-S+(\operatorname{tr} L)^{2}-\operatorname{tr}\left(L^{2}\right)+\frac{\lambda}{\tau} u=\frac{-E+2 \lambda}{\tau} .
$$

Substituting this equation into (3.11) gives

$$
\dot{L}+(\operatorname{tr} L) L-\dot{u} L-r=-\left(\frac{\lambda}{2 \tau}\right) I,
$$

which becomes Eq. (2.3) if we set $\tau=1$ and $\lambda=-\epsilon$. We have therefore deduced the following.

Theorem 5. Given a principal orbit $G / K$ where $G$ is a compact Lie group and $K$ a closed subgroup, consider on the symplectic manifold $\left(S_{+}^{2}(\mathfrak{p})^{K} \times \mathbb{R}\right) \times\left(S^{2}\left(\mathfrak{p}^{*}\right)^{K} \times \mathbb{R}^{*}\right)$ the Hamiltonian $\mathcal{H}$ given by (3.6) with $\tau=1$ and $\lambda=-\epsilon$. The integral curves of $\mathcal{H}$ lying in the variety $\{\mathcal{H}=0\}$ correspond (under the inverse Legendre transformation) to solutions of the non-mixed parts of the cohomogeneity one gradient Ricci soliton equations (2.3) and (2.4). 
Recall that by Proposition 3.19 in Ref. 12, as long as there is a singular orbit of dimension strictly less than that of the principal orbit and we can further establish $C^{3}$ regularity of the metric $\bar{g}=d t^{2}+g_{t}$ and potential $u$, then the mixed parts of the cohomogeneity one GRS equation automatically hold. In this sense the cohomogeneity one GRS equation can be viewed as a constrained Hamiltonian system.

In the remainder of this section we will derive a more explicit form of the Hamiltonian $\mathcal{H}$ in the special case where the isotropy representation of $G / K$ splits into pairwise inequivalent irreducible $\mathbb{R}$-subrepresentations.

To this end let us write

$$
\mathfrak{p}=\mathfrak{p}_{1} \oplus \cdots \oplus \mathfrak{p}_{r}
$$

for the decomposition of $\mathfrak{p}$ into $\operatorname{Ad}(K)$-irreducible $Q$-orthogonal summands and let $d_{i}=\operatorname{dim}_{\mathbb{R}} \mathfrak{p}_{i}$, so that $n=\sum_{i} d_{i}$. We will abuse notation and denote the metric endomorphism $q \in S_{+}^{2}(\mathfrak{p})^{K}$ by the diagonal operator $\operatorname{diag}\left(e^{q_{1}} I_{d_{1}}, \ldots, e^{q_{r}} I_{d_{r}}\right)$ where $I_{d_{i}}$ is the identity operator in $\operatorname{End}\left(\mathfrak{p}_{i}\right)$. In other words, via these new coordinates, we have a diffeomorphism $S_{+}^{2}(\mathfrak{p})^{K} \approx \mathbb{R}^{r}$ which in turn induces a canonical transformation of $T^{*} \mathcal{C}$, leaving the remaining variables $u, \phi$ unchanged. By abuse of notation we shall let $p_{i}$ denote the new conjugate momenta. It will be useful to let $q, p, d$ denote the vectors in $\mathbb{R}^{r}$ whose coordinates are, respectively, $q_{i}, p_{i}, d_{i}$.

Then, as in the Einstein case, we have $v=\exp \left(\frac{1}{2} d \cdot q\right)$,

$$
\operatorname{tr} L=\frac{1}{2} d \cdot \dot{q}, \quad \operatorname{tr}\left(L^{2}\right)=\frac{1}{4} \sum_{i} d_{i} \dot{q}_{i}^{2},
$$

and

$$
S=\sum_{w \in \mathcal{W}} A_{w} e^{w \cdot q}
$$

for a finite subset $W \subset \mathbb{R}^{r}$ of weight vectors and nonzero real constants $A_{w}$ which depend only on $G / K$. (See Sec. 1 in Ref. 9 for further information about $\mathcal{W}$ and $A_{w}$.)

With the above change of coordinates, the Lagrangian (3.3) becomes

$$
\mathcal{L}=e^{-u+\frac{1}{2} d \cdot q}\left(\frac{1}{4}(d \cdot \dot{q})^{2}-\frac{1}{4} \sum_{i} d_{i} \dot{q}_{i}^{2}+\dot{u}^{2}-\dot{u}(d \cdot \dot{q})+\sum_{w \in \mathcal{W}} A_{w} e^{w \cdot q}+E\right) .
$$

The Legendre transformation is now given by

$$
p_{j}=\mathcal{L}_{\dot{q}_{j}}=e^{-u+\frac{1}{2} d \cdot q}\left(\frac{1}{2}(d \cdot \dot{q}) d_{j}-\frac{1}{2} d_{j} \dot{q}_{j}-\dot{u} d_{j}\right)
$$

and

$$
\phi=\mathcal{L}_{\dot{u}}=e^{-u+\frac{1}{2} d \cdot q}(2 \dot{u}-d \cdot \dot{q}) .
$$

Using the above equations, we easily deduce that

$$
\begin{gathered}
\frac{1}{2} d \cdot \dot{q}=-\left(\sum_{j} p_{j}+\frac{n}{2} \phi\right) e^{u-\frac{1}{2} d \cdot q}, \\
\dot{u}=-\left(\sum_{j} p_{j}+\frac{n-1}{2} \phi\right) e^{u-\frac{1}{2} d \cdot q}, \\
\frac{1}{4} \sum_{i} d_{i} \dot{q}_{i}^{2}=\left(\sum_{i} \frac{p_{i}^{2}}{d_{i}}+\phi \sum_{i} p_{i}+\frac{n}{4} \phi^{2}\right) e^{2 u-d \cdot q} .
\end{gathered}
$$

Substituting the above relations in $\mathcal{H}=p(\dot{q})+\phi(\dot{u})-\mathcal{L}$ we obtain an explicit formula for $\mathcal{H}$ in the multiplicity free case.

Proposition 6. Let $G / K$ be a principal orbit whose isotropy representation splits into pairwise inequivalent irreducible summands. In terms of the exponential coordinates introduced above, 
Hamiltonian (3.6) takes the form

$\mathcal{H}=-\frac{e^{u-\frac{1}{2} d \cdot q}}{\tau}\left(\sum_{i} \frac{p_{i}^{2}}{d_{i}}+\phi \sum_{i} p_{i}+\frac{(n-1)}{4} \phi^{2}\right)+e^{-u+\frac{1}{2} d \cdot q}\left(-E+\lambda(n+1-u)-\tau \sum_{w \in \mathcal{W}} A_{w} e^{w \cdot q}\right)$.

Remark 7. The above proposition is the analogue of Proposition 2.5 in Ref. 7. The quadratic form

$$
J(p, \phi):=-\sum_{i} \frac{p_{i}^{2}}{d_{i}}-\phi \sum_{i} p_{i}-\frac{(n-1)}{4} \phi^{2}
$$

assumes a different form from its analogue in Ref. 7 , but it is still of Lorentz signature $(1, r)$. It is negative definite on the hyperplane $\phi=0$ but is positive on the vector $(d,-2)$ (in fact $J(d,-2)=1$ ).

The associated bilinear form is

$$
-\left(\sum_{i=1}^{r} \frac{p_{i} p_{i}^{\prime}}{d_{i}}+\frac{\phi}{2} \sum_{i=1}^{r} p_{i}^{\prime}+\frac{\phi^{\prime}}{2} \sum_{i=1}^{r} p_{i}+\frac{n-1}{4} \phi \phi^{\prime}\right) .
$$

It is sometimes convenient to rewrite the Hamiltonian using the extended vectors

$$
\begin{aligned}
\mathbf{d} & :=(d,-2)=\left(d_{1}, \ldots, d_{r},-2\right), \\
\mathbf{q} & :=(q, u)=\left(q_{1}, \ldots, q_{r}, u\right), \\
\mathbf{p} & :=(p, \phi)=\left(p_{1}, \ldots, p_{r}, \phi\right) .
\end{aligned}
$$

Similarly the weight vectors $w$ can be extended to $\mathbf{w}=(w, 0)$, and we will continue to use $\mathcal{W}$ to denote the set of extended vectors in $\mathbb{R}^{r+1}$. The Hamiltonian now becomes

$$
\mathcal{H}=\frac{e^{-\frac{1}{2} \mathbf{d} \cdot \mathbf{q}}}{\tau} J(\mathbf{p})+e^{\frac{1}{2} \mathbf{d} \cdot \mathbf{q}}\left(-E+\lambda(n+1-u)-\tau \sum_{w \in \mathcal{W}} A_{w} e^{\mathbf{w} \cdot \mathbf{q}}\right),
$$

where, as above,

$$
J(\mathbf{p})=-\left(\sum_{i} \frac{p_{i}^{2}}{d_{i}}+\phi \sum_{i} p_{i}+\frac{(n-1)}{4} \phi^{2}\right) .
$$

In the steady case $(\lambda=0)$, if we also set $\tau=1$, we get

$$
\mathcal{H}=e^{-\frac{1}{2} \mathbf{d} \cdot \mathbf{q}} J(\mathbf{p})-e^{\frac{1}{2} \mathbf{d} \cdot \mathbf{q}}\left(E+\sum_{w \in \mathcal{W}} A_{w} e^{\mathbf{w} \cdot \mathbf{q}}\right) .
$$

It is also often useful to enlarge the set $\mathcal{W}$ to $\tilde{W}=\mathcal{W} \cup\{0\}$, so that the final bracket in Eq. (3.15) is viewed as a sum of exponentials over $\tilde{W}$, with $E$ playing the role of $A_{0}$.

\section{CONSERVED QUANTITIES}

As in the Einstein case we can look for quantities $F$ which are generalised first integrals, in the sense that

$$
\{F, \mathcal{H}\}=\Phi \mathcal{H}
$$

so that for the soliton equations, that is, the Hamiltonian flow in the variety $\mathcal{H}=0$, the quantity $F$ is conserved. Functions in the ideal generated by $\mathcal{H}$ will be referred to as trivial generalised first integrals.

We look for solutions to (4.1) of the form

$$
F=\sum_{\mathbf{b}} F_{\mathbf{b}} e^{\mathbf{b} \cdot \mathbf{q}}: \quad \Phi=\sum_{\mathbf{b}} \Phi_{\mathbf{b}} e^{\mathbf{b} \cdot \mathbf{q}},
$$

where $F_{\mathbf{b}}$ and $\Phi_{\mathbf{b}}$ are polynomials in $\mathbf{p}$. In view of this we shall in this section denote by $\nabla_{\mathbf{p}}$ the gradient operator in the momentum variables whenever the possibility of confusion may occur. In 
cases where the operator is applied to functions such as $J, F$, and $\Phi$ which depend solely on $\mathbf{p}$ we shall suppress this subscript.

Substituting these into (4.1) and setting $\psi:=\Phi-\frac{1}{2} \mathbf{d} \cdot \nabla_{\mathbf{p}} F$ we obtain, upon using (3.15), the recursion relation

$$
(\mathbf{b} \cdot \nabla J) F_{\mathbf{b}}-\psi_{\mathbf{b}} J=-E\left(\psi_{\mathbf{b}-\mathbf{d}}+\mathbf{d} \cdot \nabla F_{\mathbf{b}-\mathbf{d}}\right)-\sum_{w} A_{w}\left(\psi_{\mathbf{b}-\mathbf{d}-\mathbf{w}}+(\mathbf{d}+\mathbf{w}) \cdot \nabla F_{\mathbf{b}-\mathbf{d}-\mathbf{w}}\right) .
$$

We adopt a similar strategy as in Ref. 7 to look for nontrivial generalised first integrals, starting with a seed level $\mathbf{c}$ where there is a factorisation

$$
J=(\mathbf{c} \cdot \nabla J) \theta
$$

so we may obtain a nontrivial solution to the recursion (i.e., one where we do not have $F_{\mathbf{c}}=J G$ and $\left.\psi_{\mathbf{c}}=(\mathbf{c} . \nabla J) G\right)$ at this level by setting

$$
F_{\mathbf{c}}=\theta \psi_{\mathbf{c}} .
$$

Let us consider the Bryant soliton where the hypersurface is just the sphere $S^{n}$, viewed as the isotropy irreducible space $S O(n+1) / S O(n)$. (In the literature, using this particularly simple form of the sphere in the cohomogeneity one ansatz is referred to as the rotationally symmetric case.) We write the soliton metric as

$$
\bar{g}=d t^{2}+h(t)^{2} g_{1}
$$

where $g_{1}$ is the constant curvature one metric on $S^{n}$. The scalar curvature function for the sphere is $n(n-1) e^{-q_{1}}$, where we set $h(t)^{2}=e^{q_{1}}$. Writing $q$ for $q_{1}$, we have

$$
\begin{gathered}
\mathbf{d}=(n,-2), \mathbf{q}=(q, u), \mathbf{p}=(p, \phi), \\
\tilde{W}=\{(0,0),(-1,0)\}
\end{gathered}
$$

(for $n>1$ ), and

$$
J=-\left(\frac{p^{2}}{n}+p \phi+\frac{n-1}{4} \phi^{2}\right) .
$$

If $n=1$ the hypersurface reduces to a circle and so $\tilde{W}=\{(0,0)\}$, i.e., $\mathcal{W}$ is empty.

We may factorise $J$ as above where

$$
\mathbf{c}=\left(-\frac{1}{2}(n+\sqrt{n}), 1\right) \text { and } \theta=-\left(\frac{p}{\sqrt{n}}+\frac{\sqrt{n}-1}{2} \phi\right) .
$$

So we have a factorisation of $J$ over the rationals if and only if $n$ is a perfect square. This is exactly the condition singled out by Painlevé analysis of the Bryant system in Ref. 3.

We begin with a simple example.

Example 8. The Bryant system with $n=1$ takes a particularly simple form, as we only have the zero vector in $\tilde{W}$.

Now $J=-p(p+\phi)$, and we obtain a factorisation with $\mathbf{c}=(-1,1)$ or $(0,1)$. Choosing the latter we have $\theta=p+\phi$. We can now start the recursion with $F_{\mathbf{c}}=\theta, \psi_{\mathbf{c}}=1$. Since

$$
\psi_{\mathbf{c}}+\mathbf{d} \cdot \nabla F_{\mathbf{c}}=1+(1,-2) \cdot(1,1)=0
$$

we have a full solution to the recursion with all other terms zero. The conserved quantity is

$$
F=(p+\phi) e^{u} .
$$

In the variables of the discussion of the Bryant system in Ref. 5 (Chapter 1, Sec. 4.1) this is just $x+y$.

In Ref. 7 (see Sec. 5), in certain situations with two weight vectors $v$ and $w$ we obtained some nontrivial conserved quantities as follows. We were able to write $F_{\mathbf{c}}$ in two different ways

$$
F_{\mathbf{c}}=J \Gamma_{v}+\tau \theta^{s^{\prime}}=J \Gamma_{w}+\rho \theta^{s},
$$


where

$$
\begin{gathered}
(\mathbf{v}+\mathbf{d}) \cdot \nabla \tau=(\mathbf{w}+\mathbf{d}) \cdot \nabla \rho=0, \\
(\mathbf{v}+\mathbf{d}) \cdot \nabla \theta=-\frac{1}{s^{\prime}}, \quad(\mathbf{w}+\mathbf{d}) \cdot \nabla \theta=-\frac{1}{s},
\end{gathered}
$$

and $\Gamma_{v}, \Gamma_{w}$ are constant. One now has a solution to the recursion with

$$
F_{\mathbf{c}+\mathbf{z}+\mathbf{d}}=-A_{z} \Gamma_{z}, \quad \psi_{\mathbf{c}+\mathbf{z}+\mathbf{d}}=0, \quad \text { with } \mathbf{z}=\mathbf{v}, \mathbf{w}
$$

and all other $F_{\mathbf{a}}, \psi_{\mathbf{a}}$ zero.

In our situation, we have $v=0$. Taking $\theta=-\left(\frac{p}{\sqrt{n}}+\frac{\sqrt{n}-1}{2} \phi\right)$, as above, we have

$$
\mathbf{d} \cdot \nabla \theta=-1, \quad(\mathbf{d}+\mathbf{w}) \cdot \nabla \theta=-1+\frac{1}{\sqrt{n}} .
$$

We see that the latter term is minus the reciprocal of an integer if and only if $n=4$, when it is $-\frac{1}{2}$.

Example 9. In the $n=4$ case we have

$$
\mathbf{d}=(4,-2), \quad \mathbf{c}=(-3,1), \quad \theta=-\frac{1}{2}(p+\phi) .
$$

We may take $\tau=(p+2 \phi)$, so that $\mathbf{d} \cdot \nabla \tau=0$. We now have

$$
-\theta^{2}=-J+\tau \theta=-\frac{1}{4}(p+\phi)^{2}
$$

so the above conditions are satisfied with $\rho=-1, \Gamma_{0}=-1, \Gamma_{w}=0$, and $s=2, s^{\prime}=1$.

We obtain a nontrivial solution to the recursion relations,

$$
\begin{aligned}
\psi_{\mathbf{c}}=\frac{1}{2}(p+\phi), & F_{\mathbf{c}}=\theta \psi_{\mathbf{c}}=-\frac{1}{4}(p+\phi)^{2}, \\
\psi_{\mathbf{c}+\mathbf{d}+\mathbf{w}} & =0, \quad F_{\mathbf{c}+\mathbf{d}+\mathbf{w}}=0, \\
\psi_{\mathbf{c}+\mathbf{d}} & =0, \quad F_{\mathbf{c}+\mathbf{d}}=E .
\end{aligned}
$$

The conserved quantity is now

$$
F=-\frac{1}{4}(p+\phi)^{2} e^{-3 q+u}+E e^{q-u}
$$

We will show in Sec. VI that this generalized first integral allows us to write down the Bryant soliton in dimension 5 explicitly.

Remark 10. It is interesting to observe that in the $n=4$ case one may also find a conserved quantity by the method of Darboux polynomials (see Refs. 18 and 14). It is convenient to use the variables of Ref. 5 (Chapter 1, Sec. 4.1), $x=\dot{h}, y=n \dot{h}-h \dot{u}$. Using as our independent variable $T$ defined by $h d T=d t,{ }^{5}$ derives the equations

$$
\begin{aligned}
& x^{\prime}=x^{2}-x y+n-1, \\
& y^{\prime}=x(y-n x) .
\end{aligned}
$$

Given a planar system

$$
x^{\prime}=P(x, y), \quad y^{\prime}=Q(x, y)
$$

representing the flow associated to the vector field $X=P \partial_{x}+Q \partial_{y}$, we call a polynomial $J$ a Darboux polynomial if $X(J)=g J$ for some polynomial $g$. Sufficiently many Darboux polynomials may be used to construct conserved quantities.

For our system we always have a Darboux polynomial $J_{1}=n x^{2}-y^{2}+n(n-1)$, for which $X\left(J_{1}\right)=2 x J_{1}$. This is associated to the soliton conservation law, which in these variables is

$$
n x^{2}-y^{2}+n(n-1)=C h^{2} \text {. }
$$


If $n=4$ there is a second Darboux polynomial, $J_{2}=2 x^{2}-x y+3$, which satisfies $X\left(J_{2}\right)=(4 x-$ y) $J_{2}$. This means that $R=\frac{\sqrt{J_{1}}}{J_{2}}$ satisfies

$$
X(R)=(-3 x+y) R=-\operatorname{div}(X) R .
$$

So $R$ is an integrating factor in the sense that $(R Q)_{y}+(R P)_{x}=0$. There is now an $I$ such that $I_{x}=R Q$ and $I_{y}=-R P$, and $I$ is a conserved quantity.

\section{SUPERPOTENTIALS}

A superpotential for a Hamiltonian is a time-independent solution to the Hamilton-Jacobi equations, i.e., a function $\mathbf{q} \mapsto f(\mathbf{q})$ on configuration space that solves the equation

$$
H(\mathbf{q}, d f(\mathbf{q}))=0 .
$$

As we shall describe in Sec. VI, superpotentials are important because they define subsystems of the full Hamiltonian system, which are often more tractable than the full system. In Refs. 8, 9, and 13 we analysed the existence of superpotentials in the Einstein case.

In the steady soliton case the above equation becomes

$$
J(\nabla f, \nabla f)=e^{\mathbf{d} \cdot \mathbf{q}}\left(E+\sum_{w \in \mathcal{W}} A_{w} e^{\mathbf{w} \cdot \mathbf{q}}\right),
$$

where $\nabla f$ denotes the Euclidean gradient with respect to the variables $\mathbf{q}$. We look for superpotentials of the form

$$
f=\sum f_{\mathbf{c}} e^{\mathbf{c} \cdot \mathbf{q}}
$$

in which $f_{\mathbf{c}}$ are constant and the sum ranges over a finite set $C$ of points $\mathbf{c}$ in $\mathbb{R}^{r+1}$. This leads to

$$
\sum_{\mathbf{a}+\mathbf{c}=\mathbf{b}} J(\mathbf{a}, \mathbf{c}) f_{\mathbf{a}} f_{\mathbf{c}}= \begin{cases}A_{w}, & \text { if } \mathbf{b}=\mathbf{d}+\mathbf{w} \text { for some } w \in \mathcal{W} \\ E, & \text { if } \mathbf{b}=\mathbf{d}, \\ 0, & \text { otherwise. }\end{cases}
$$

The following lemma is often useful.

Lemma 11. Let $\mathbf{v}, \mathbf{w}$ be vectors in $\mathbb{R}^{r+1}$ with $v_{r+1}=w_{r+1}=0$. Then

$$
J(\mathbf{v}+\mathbf{d}, \mathbf{w}+\mathbf{d})=1-\sum_{i=1}^{r} \frac{v_{i} w_{i}}{d_{i}} .
$$

Proof. By (3.14),

$$
\begin{aligned}
J(\mathbf{d}+\mathbf{v}, \mathbf{d}+\mathbf{w}) & =-\left(\sum_{i=1}^{r} \frac{\left(d_{i}+v_{i}\right)\left(d_{i}+w_{i}\right)}{d_{i}}-\sum_{i=1}^{r}\left(d_{i}+v_{i}\right)-\sum_{i=1}^{r}\left(d_{i}+w_{i}\right)+\left(\frac{n-1}{4}\right)(-2)^{2}\right) \\
& =-\left(\sum_{i=1}^{r}\left(\frac{v_{i} w_{i}}{d_{i}}-d_{i}\right)+n-1\right) \\
& =1-\sum_{i=1}^{r} \frac{v_{i} w_{i}}{d_{i}} .
\end{aligned}
$$

We can deduce from (5.3) the following result, whose proof is essentially the same to that in the Einstein case (see Propositions 2.2-2.6 of Ref. 8). We use $\operatorname{conv}(S)$ to denote the convex hull of a set $S$. As mentioned earlier, we will identify $\tilde{W}$ with a set in $\mathbb{R}^{r+1}$ by associating to each weight vector $w$ the extended vector $\mathbf{w}=(w, 0)$ in $\mathbb{R}^{r+1}$. 
Lemma 12. (i) The convex hull of $C$ contains the convex hull of $\frac{1}{2}(\mathbf{d}+\tilde{W})$.

(ii) If $\mathbf{a}, \mathbf{c} \in C$ and $\mathbf{a}+\mathbf{c}$ cannot be written as the sum of two elements of $C$ distinct from $\mathbf{a}, \mathbf{c}$, then either $J(\mathbf{a}, \mathbf{c})=0$ or $\mathbf{a}+\mathbf{c} \in \mathbf{d}+\tilde{\mathcal{W}}$. In particular, if $\mathbf{c}$ is a vertex of $\operatorname{conv}(C)$, then either $\mathbf{c}$ is $J$-null or $2 \mathbf{c}=\mathbf{d}+\mathbf{w}$ for some $w \in \tilde{W}$ and $J(\mathbf{c}, \mathbf{c}) f_{\mathbf{c}}^{2}=A_{w}$.

If we further assume that no vertex of $\operatorname{conv}(C)$ is $J$-null, then we also have the following:

(iii) The convex hull of $C$ equals the convex hull of $\frac{1}{2}(\mathbf{d}+\tilde{W})$. In particular every element of $C$ is of the form $\mathbf{c}=\frac{1}{2}(\mathbf{d}+\mathbf{x})$ for $\mathbf{x} \in \mathbb{R}^{r+1}$ with $-1 \leq \sum x_{i} \leq 0$. Moreover, $\sum x_{i}=-1$ if and only if $\mathbf{x}$ is a convex linear combination of nonzero elements of $\tilde{W}$ (i.e., of elements of $\mathcal{W}$ ). Also $\sum x_{i}=0$ if and only if $\mathbf{x}=0$, that is, $\mathbf{c}=\frac{1}{2} \mathbf{d}$.

(iv) If $\mathbf{w}$ is a vertex of $\operatorname{conv}(\tilde{\mathcal{W}})$ then $\mathbf{w}+\mathbf{d}=2 \mathbf{c}$ for some vertex $\mathbf{c}$ of $\operatorname{conv}(C)$. Moreover $J(\mathbf{d}+\mathbf{w}, \mathbf{d}+\mathbf{w})$ has the same sign as $A_{w}$.

If we make the non-null vertex assumption in the second half of the above lemma, we can deduce a non-existence result for superpotentials, using similar arguments to the result in the Einstein case for nonzero cosmological constant (cf. Theorem 10.1 of Ref. 8).

Proposition 13. If no vertex of $\operatorname{conv}(C)$ is $J$-null, then there are no superpotentials of form (5.2).

Proof. Let $\mathbf{w}$ be a vertex of $\operatorname{conv}(\mathcal{W})$, so the line segment $0 \mathbf{w}$ is an edge of $\operatorname{conv}(\tilde{\mathcal{W}})$. The preceding Lemma 12 shows that $\mathbf{c}_{0}=\frac{1}{2} \mathbf{d}$ and $\mathbf{c}_{1}=\frac{1}{2}(\mathbf{d}+\mathbf{w})$ are vertices of $\operatorname{conv}(C)$ and $\mathbf{c}_{0} \mathbf{c}_{1}$ is an edge of $\operatorname{conv}(C)$.

Lemma 11 shows that $J\left(\mathbf{c}_{0}, \mathbf{c}_{0}\right)=\frac{1}{4} J(\mathbf{d}, \mathbf{d})=\frac{1}{4}$, and also $J\left(\mathbf{c}_{0}, \mathbf{c}_{1}\right)=\frac{1}{4} J(\mathbf{d}, \mathbf{d}+\mathbf{w})=\frac{1}{4}$. As $J\left(\mathbf{c}_{0},.\right)$ is an affine function on the edge $\mathbf{c}_{0} \mathbf{c}_{1}$, it is therefore constant.

By considering the element of $C$ on $\mathbf{c}_{0} \mathbf{c}_{1}$ which is distinct from $\mathbf{c}_{0}$ and closest to $\mathbf{c}_{0}$ and applying part (ii) of Lemma 12, we obtain a contradiction.

Remark 14. It is interesting to note that in the Ricci-flat case there are several superpotentials satisfying the non-null assumption (see the discussion in Ref. 8, especially Theorem 6.1). So the above result is another manifestation of the greater rigidity of the soliton equations compared to the Ricci-flat Einstein equations.

On the other hand, if we relax the non-null assumption, we can find some examples of superpotentials in the steady case.

Example 15. We first consider the situation of the Bryant soliton for which $\tilde{W}=\{(0,0)$, $(-1,0)\}$ if $n>1$.

We observe using Lemma 11 that the only null vectors with zero second component are $\pm(\sqrt{n}, 0)$. In particular if $n=4$ then we can take $C$ to consist of the vectors $\frac{1}{2} \mathbf{d}$ and $\frac{1}{2}(\mathbf{d}+(-2,0))$, where $\mathbf{d}=(4,-2)$. The first gives us $\mathbf{d}+(0,0)$ in the expansion of $J(\nabla f, \nabla f)$, the cross term gives $\mathbf{d}+(-1,0)$, and the last vector is null so does not contribute. We therefore obtain a superpotential

$$
f=2 \sqrt{E} e^{2 q_{1}-u}+\frac{12}{\sqrt{E}} e^{q_{1}-u}
$$

for positive $E$.

When $n=1$, we can obtain a superpotential in another way because the extended vectors $\mathbf{d}+( \pm 1,0)$ are both $J$-null. We let $C$ consist of the vectors $\mathbf{c}_{1}=(1,-1)$ and $\mathbf{c}_{2}=(0,-1)$. Note that the scalar curvature function is 0 , so the parts of the superpotential equation involving $\mathbf{c}_{i}+\mathbf{c}_{i}$ automatically hold. The single cross term leads to the condition $f_{1} f_{2}=E$. So if $E \neq 0$ we get the superpotential

$$
a e^{q_{1}-u}+\frac{E}{a} e^{-u}, a \neq 0
$$

while if $E=0$ we get for $a \neq 0$ we get the possibilities

$$
f=a e^{q_{1}-u} \text { and } f=a e^{-u} .
$$

These will be referred to as the limiting cases of the previous superpotential. 
Example 16. Next we consider the case of a warped product on two factors, so that the metric is

$$
\bar{g}=d t^{2}+h_{1}(t)^{2} g_{1}+h_{2}(t)^{2} g_{2}
$$

where $\left(M_{i}, g_{i}\right)$ is Einstein of dimension $d_{i}$ with positive Einstein constant normalised to be $d_{i}-1$.

Now $r=2$ and $\tilde{W}=\{(0,0,0),(-1,0,0),(0,-1,0)\}$. We pick $C$ to consist of vectors

$$
\begin{aligned}
& \mathbf{c}_{1}=\frac{1}{2}(\mathbf{d}+(-1,-1,0)), \\
& \mathbf{c}_{2}=\frac{1}{2}(\mathbf{d}+(-1,1,0)), \\
& \mathbf{c}_{3}=\frac{1}{2}(\mathbf{d}+(1,-1,0)) .
\end{aligned}
$$

Now the cross terms give us the elements of $\tilde{W}$, and $J\left(\mathbf{c}_{i}, \mathbf{c}_{j}\right)$ is positive for $i \neq j$. If $E$ is positive, we may therefore obtain a superpotential provided that $\mathbf{c}_{1}, \mathbf{c}_{2}, \mathbf{c}_{3}$ are null, which translates into the condition $\frac{1}{d_{1}}+\frac{1}{d_{2}}=1$. Therefore, if $\left(d_{1}, d_{2}\right)=(2,2)$, we obtain a superpotential given by

$$
F=\sqrt{E} e^{\frac{1}{2} q_{1}+\frac{3}{2} q_{2}-u}+\sqrt{E} e^{\frac{3}{2} q_{1}+\frac{1}{2} q_{2}-u}+\frac{4}{\sqrt{E}} e^{\frac{1}{2} q_{1}+\frac{1}{2} q_{2}-u} .
$$

Remark 17. In the $n=4$ case of Example 15 and in Example 16, the superpotential condition forces $E$ to be positive. Recalling from the discussion preceding Proposition 3 that in the steady case $E$ is just equal to minus the constant $C$, it is interesting to observe that the condition $E>0$ is also a consequence of assuming completeness of the metric (cf. Ref. 4).

Example 18. This example involves the Bérard-Bergery-Calabi ansatz, that is, we take the hypersurface to be a circle bundle over a product of $r \geq 1$ Fano Kähler-Einstein manifolds of real dimension $d_{i}=2 m_{i}$. Thus $\mathbf{d}=\left(1,2 m_{2}, \ldots, 2 m_{r},-2\right)$.

Now $\tilde{W}$ consists of

(i) type III vectors with 1 in the first place and -2 in the $i$ th place for $2 \leq i \leq r$,

(ii) type I vectors with -1 in $i$ th place $(2 \leq i \leq r)$, and

(iii) the zero vector.

For $C$, we take

$$
\mathbf{c}_{1}=\frac{1}{2}(\mathbf{d}+(-1,0, \ldots, 0))
$$

$\mathbf{c}_{2}, \ldots, \mathbf{c}_{r}$ to be $\frac{1}{2}(\mathbf{d}+\mathbf{w})$ where $\mathbf{w}$ ranges over the type III vectors, and

$$
\mathbf{c}_{r+1}=\frac{1}{2}(\mathbf{d}+(1,0, \ldots, 0)) \text {. }
$$

As $d_{1}=1$, we see that $\mathbf{c}_{1}$ and $\mathbf{c}_{r+1}$ are null, while $\mathbf{c}_{i}:(2 \leq i \leq r)$ are mutually orthogonal, and also orthogonal to $\mathbf{c}_{r+1}$. The cross terms $\mathbf{c}_{1}+\mathbf{c}_{i}$ for $2 \leq i \leq r$ give the type I vectors in $\mathbf{d}+\tilde{\mathcal{W}}$, while $2 \mathbf{c}_{i}$ give the type III vectors and $\mathbf{c}_{1}+\mathbf{c}_{r+1}$ gives the zero vector.

We let $A_{i}$ for $i=2, \ldots, r$ denote the constants $A_{\mathrm{w}}$ for the type III vectors, and $A_{r+i-1}$ denote the constants for the corresponding type I vectors. We must take $f_{i}=\sqrt{-A_{i} d_{i}}$ for $2 \leq i \leq r$ (possible since $A<0$ for type III vectors). We see finally that we obtain a superpotential provided $A_{r+i-1} / \sqrt{-A_{i} d_{i}}$ is the same for each $2 \leq i \leq r$.

If we normalize the Kähler-Einstein metric on $M_{i}$ so that its Kähler class equals $2 \pi \alpha_{i}$ where $\alpha_{i}$ is the indivisible integral cohomology class so that the first Chern class of $M_{i}$ is $\kappa_{i} \alpha_{i}$ with $\kappa_{i}>0$, then $A_{r+i-1}=d_{i} \kappa_{i}$. Also, suppose that the Euler class of the circle bundle is $b_{2} \alpha_{2}+\cdots+b_{r} \alpha_{r}$. Then it follows that $A_{i}=-\frac{1}{4} d_{i} b_{i}^{2}$ for $2 \leq i \leq r$. The conditions guaranteeing a superpotential in the previous paragraph translate into requiring $\kappa_{i} /\left|b_{i}\right|$ to be independent of $i$. Note that this is precisely the condition under which an explicit, complete, steady Kähler Ricci soliton was constructed in Ref. 12 (see Theorem 4.20(i) in that reference).

Note furthermore that unlike the previous examples, there is no constraint on the sign of $E$ here. 


\section{EXPLICIT NON-KÄHLER STEADY SOLITONS}

One reason for the importance of superpotentials is that they give rise to a Lagrangian section of the cotangent bundle of configuration space that is invariant under the Hamiltonian flow. Using this section to pull back the Hamiltonian vector field to configuration space, one obtains a first order subsystem of the canonical equations (see Sec. 1 of Ref. 8 for details). For the Hamiltonian system associated to the cohomogeneity one gradient Ricci soliton equation, the first order subsystem is given by

$$
\dot{\mathbf{q}}=2 \mathbf{v}^{-1} J \nabla f,
$$

where $\mathbf{v}$ is the extended relative volume $e^{\frac{1}{2} \mathbf{d} \cdot \mathbf{q}}=v e^{-u}$ and $f$ is the superpotential.

In the following we shall consider the first order subsystems for Examples 15 and 16 and show that they lead to explicit solutions of the soliton equation. Explicit solutions arising from the superpotentials in Example 18 were discussed in Sec. 4 of Ref. 12.

For Example 15 with $n=4$ we have

$$
J=-\left(\begin{array}{ll}
\frac{1}{4} & \frac{1}{2} \\
\frac{1}{2} & \frac{3}{4}
\end{array}\right),
$$

so Eq. (6.1) and the superpotential constructed in that example yield the system

$$
\begin{aligned}
& \dot{q}_{1}=\frac{6}{\sqrt{E}} e^{-q_{1}}, \\
& \dot{u}=-\sqrt{E}+\frac{6}{\sqrt{E}} e^{-q_{1}} .
\end{aligned}
$$

Since $h(t)^{2}=e^{q_{1}}$, we have $\frac{d}{d t} h^{2}=\frac{6}{\sqrt{E}}$, and so

$$
\begin{aligned}
& h(t)=\frac{\sqrt{6}}{E^{1 / 4}} \sqrt{t+t_{0}}, \\
& u(t)=-\sqrt{E} t+\log \left(t+t_{0}\right)+\text { const }
\end{aligned}
$$

where $t_{0}$ is a constant.

Notice that there is no smooth soliton among this one-parameter family of solutions. Assuming that we place the singular orbit at $t=0$, the smoothness conditions require $h(0)=0$, so that $t_{0}=0$. But we also need $\dot{h}(0)=1$, which is never satisfied. However, the solitons are complete at infinity.

Remark 19. The above family of explicit singular solitons was first constructed in Ref. 1 (see Proposition 2.2 and Remark 2.6). They belong to a family of such solitons which occur in all dimensions $\geq 3$. For general dimensions, the solitons were constructed by dynamical systems methods. The existence of a superpotential helps to explain why dimension 5 is special.

In the $n=1$ case of Example 15 one easily checks that the first superpotential gives rise to the first order system

$$
\begin{aligned}
& \dot{q}_{1}=-a e^{\frac{1}{2} q_{1}}+\left(\frac{E}{a}\right) e^{-\frac{1}{2} q_{1}}, \\
& \dot{u}=-a e^{\frac{1}{2} q_{1}} .
\end{aligned}
$$

Note that the first equation above can be expressed in terms of $h$ as

$$
\dot{h}=\frac{a}{2}\left(\frac{E}{a^{2}}-h^{2}\right),
$$

which is precisely of the form of Eq. (1.42) in Chapter I of Ref. 5. The point is that this equation follows immediately from the existence of a superpotential and is not the consequence of ad hoc derivations. We will skip the detailed analysis of the associated first order system since 
2-dimensional gradient Ricci solitons, singular or otherwise, have been classified in detail in Ref. 2. However, we do want to point out the solutions of the first order system yield all the families of steady solitons given in Ref. 2. In other words, all 2-dimensional steady gradient Ricci solitons, singular or not, have an associated conserved quantity that is linear in momentum.

We also note that the $h$ identically constant case (necessarily with $E>0$ ) gives the cylinder, and the 1-parameter family of cigar solitons (necessarily with $E>0$ ) can be characterised as members of a 2-parameter family of generically singular solitons which also satisfy the smoothness condition $E=2 a$.

The two limiting cases of the superpotential give rise, respectively, to singular solitons which are the limits (as $E / a$ tends to 0 ) of the family of "exploding solitons," and a family of flat cone solutions, which include Euclidean space as a special case.

In Example 16, we found a superpotential when $d_{1}=d_{2}=2$. The matrix for $J$ is therefore

$$
J=-\left(\begin{array}{ccc}
\frac{1}{2} & 0 & \frac{1}{2} \\
0 & \frac{1}{2} & \frac{1}{2} \\
\frac{1}{2} & \frac{1}{2} & \frac{3}{4}
\end{array}\right) .
$$

Hence with $h_{1}^{2}=e^{q_{1}}$ and $h_{2}^{2}=e^{q_{2}}$, the associated first order subsystem is

$$
\begin{aligned}
& \dot{q}_{1}=\frac{\sqrt{E}}{2} e^{-\frac{1}{2} q_{1}+\frac{1}{2} q_{2}}-\frac{\sqrt{E}}{2} e^{\frac{1}{2} q_{1}-\frac{1}{2} q_{2}}+\frac{2}{\sqrt{E}} e^{-\frac{1}{2} q_{1}-\frac{1}{2} q_{2}}, \\
& \dot{q}_{2}=-\frac{\sqrt{E}}{2} e^{-\frac{1}{2} q_{1}+\frac{1}{2} q_{2}}+\frac{\sqrt{E}}{2} e^{\frac{1}{2} q_{1}-\frac{1}{2} q_{2}}+\frac{2}{\sqrt{E}} e^{-\frac{1}{2} q_{1}-\frac{1}{2} q_{2}}, \\
& \dot{u}=-\frac{\sqrt{E}}{2} e^{-\frac{1}{2} q_{1}+\frac{1}{2} q_{2}}-\frac{\sqrt{E}}{2} e^{\frac{1}{2} q_{1}-\frac{1}{2} q_{2}}+\frac{2}{\sqrt{E}} e^{-\frac{1}{2} q_{1}-\frac{1}{2} q_{2}} .
\end{aligned}
$$

The first two equations imply that

$$
\operatorname{tr} L=2\left(\frac{\dot{h_{1}}}{h_{1}}+\frac{\dot{h_{2}}}{h_{2}}\right)=\frac{4}{\sqrt{E}} \frac{1}{h_{1} h_{2}} .
$$

So $h_{1} h_{2}=\frac{2}{\sqrt{E}}\left(t+t_{0}\right)$ for some constant $t_{0}$. Using this relation in Eq. (6.3), after some simplification, we obtain

$$
\frac{d}{d t}\left(h_{1}^{2}\right)=\left(t+t_{0}\right)-\frac{E}{4\left(t+t_{0}\right)} h_{1}^{4}+\frac{h_{1}^{2}}{t+t_{0}} .
$$

Let us set $\beta:=\frac{h_{1}^{2}}{t+t_{0}}$. Then $\beta$ satisfies

$$
4 \dot{\beta}=4-E \beta^{2},
$$

which again is analogous to Eq. (6.2)

A special solution of this equation is $\beta= \pm \frac{2}{\sqrt{E}}$, which gives

$$
h_{1}(t)^{2}= \pm \frac{2}{\sqrt{E}}\left(t+t_{0}\right) .
$$

Note that the corresponding metrics cannot be smooth.

If we take $\sqrt{E} \beta<2$, we obtain

$$
\beta=\left(\frac{2}{\sqrt{E}}\right) \frac{e^{\sqrt{E}\left(t+t_{1}\right)}-1}{e^{\sqrt{E}\left(t+t_{1}\right)}+1},
$$

where $t_{1}$ is a constant. This in turn yields

$$
h_{1}(t)^{2}=\left(\frac{2}{\sqrt{E}}\right)\left(t+t_{0}\right) \frac{e^{\sqrt{E}\left(t+t_{1}\right)}-1}{e^{\sqrt{E}\left(t+t_{1}\right)}+1},
$$




$$
h_{2}(t)^{2}=\left(\frac{2}{\sqrt{E}}\right)\left(t+t_{0}\right) \frac{e^{\sqrt{E}\left(t+t_{1}\right)}+1}{e^{\sqrt{E}\left(t+t_{1}\right)}-1},
$$

and

$$
\dot{u}=-\sqrt{E}+\frac{1}{t+t_{0}}-\frac{2 \sqrt{E}}{e^{2 \sqrt{E}\left(t+t_{1}\right)}-1} .
$$

Let us now look for complete smooth solutions within this 3-parameter family of solutions. We need to have $h_{1}(0)=0, \dot{h_{1}}(0)=1, \dot{u}(0)=0$, and $h_{2}(0)>0, \dot{h_{2}}(0)=0$. Hence $t_{0}=t_{1}=0$, and one easily checks that these choices imply that all smoothness conditions at $t=0$ (the position of the zero section) hold. We therefore obtain the following 1-parameter family of explicit solutions:

$$
\begin{gathered}
h_{1}(t)=\frac{\sqrt{2 t}}{E^{1 / 4}}\left(\tanh \left(\frac{t \sqrt{E}}{2}\right)\right)^{1 / 2}, \quad h_{2}(t)=\frac{\sqrt{2 t}}{E^{1 / 4}}\left(\operatorname{coth}\left(\frac{t \sqrt{E}}{2}\right)\right)^{1 / 2}, \\
u(t)=\log \left(\frac{t \sinh (\sqrt{E})}{\sinh (\sqrt{E} t)}\right)+\text { const. }
\end{gathered}
$$

It follows that the mean curvature of the hypersurfaces is $\operatorname{tr} L=\frac{2}{t}$ and the scalar curvature of the soliton metric is

$$
\bar{R}=\frac{2 \sqrt{E}}{t}-\frac{1}{t^{2}}-\frac{4 \sqrt{E}}{e^{2 \sqrt{E} t}-1}\left(\sqrt{E}-\frac{1}{t}\right)-\frac{4 E}{\left(e^{2 \sqrt{E} t}-1\right)^{2}} .
$$

Similarly, we can analyse solutions for which $2<\sqrt{E} \beta$ holds. Now we have

$$
h_{1}(t)=\frac{\sqrt{2}}{E^{1 / 4}} \sqrt{t+t_{0}}\left(\operatorname{coth}\left(\frac{\sqrt{E}}{2}\left(t+t_{1}\right)\right)\right)^{1 / 2}
$$

and we obtain smooth complete solutions in which the $h_{1}, h_{2}$ in the case treated above are swapped.

Finally, we will use the generalized first integral (4.3) we found for the Bryant soliton system (Example 9) in dimension 5 to obtain an explicit expression for the Bryant soliton in that dimension.

Using the Legendre transformation formulae of Sec. III, we can rewrite (4.3) as

$$
F=e^{-u} h^{2}\left(E-\left(\frac{2 \dot{h}}{h}-\dot{u}\right)^{2}\right) .
$$

We note first that the conservation law $F=\mu$ can be rewritten in terms of a new variable $\beta$ := $-\log \left(\frac{e^{u}}{h^{2}}\right)$ as

$$
(\dot{\beta})^{2}=E-\mu e^{-\beta}
$$

which can be integrated explicitly. The Hamiltonian constraint becomes

$$
-(\dot{\beta})^{2}-4 \frac{\dot{\beta} \dot{h}}{h}+E+\frac{12}{h^{2}}=0
$$

Setting $\alpha=h^{2}$ we have the linear equation in $\alpha$,

$$
2 \dot{\beta} \dot{\alpha}-\mu e^{-\beta} \alpha=12,
$$

so the system has been integrated by quadratures. We recover the soliton potential via

$$
u=-\beta+\log \alpha .
$$

In order to obtain the Bryant soliton, we assume $\mu<0$ and choose

$$
\dot{\beta}=\sqrt{E-\mu e^{-\beta}} .
$$

Upon integration we get

$$
\beta=-\log \left(\frac{E}{-\mu}\left(\operatorname{coth}^{2}\left(\frac{\sqrt{E}}{2}\left(t+t_{0}\right)\right)-1\right)\right),
$$


where $t_{0}$ is a constant. Substituting this into the first order equation for $\alpha$ results in

$$
\alpha=h(t)^{2}=\frac{6}{\sqrt{E}}\left(\left(t+t_{0}+t_{1}\right) \operatorname{coth}\left(\frac{\sqrt{E}}{2}\left(t+t_{0}\right)\right)-\frac{2}{\sqrt{E}}\right)
$$

for some integration constant $t_{1}$.

The smoothness conditions $h(0)=0$ and $\dot{h}(0)=1$ are then easily seen to be satisfied if we choose $t_{0}=t_{1}=0$. The soliton potential is given (up to an additive constant) by

$$
u=\log \left(\left(\operatorname{coth}^{2}\left(\frac{\sqrt{E}}{2} t\right)-1\right)\left(t \operatorname{coth}\left(\frac{\sqrt{E}}{2} t\right)-\frac{2}{\sqrt{E}}\right)\right) .
$$

Remark 20. (a) If we instead choose $t_{0}=0$ and $t_{1}>0$ in the above, we obtain a 1-parameter family of solutions which are complete at $\infty$ but which blow up like $\frac{1}{t}$ at the origin.

(b) If we choose $\mu$ to be 0 in the conservation law above, we recover the steady solitons with a conical singularity at $t=0$ associated with superpotential (5.5).

(c) We can also take $\mu$ to be positive. In this case we obtain a 1-parameter family of solutions which are complete at $\infty$ such that $h(0)=0$ and $\dot{h}(0)=+\infty$. In fact

$$
h(t)^{2}=\alpha=\frac{6}{\sqrt{E}}\left(\left(t+t_{0}\right) \tanh \left(\frac{\sqrt{E}}{2}\left(t+t_{0}\right)\right)-\frac{2}{\sqrt{E}}\right),
$$

where $t_{0}(E)>0$ is the unique positive solution of $\frac{\sqrt{E}}{2} t_{0} \tanh \left(\frac{\sqrt{E}}{2} t_{0}\right)=1$.

(d) We certainly expect that singular solitons with the properties of (a) and (c) above exist in all dimensions. As mentioned before (see Remark 19), ones with properties in (b) were found in Ref. 1.

\section{ACKNOWLEDGMENTS}

A. Betancourt is supported by the Mexican Council of Science and Technology (CONACyT). M. Wang is partially supported by NSERC Grant No. OPG0009421.

${ }^{1}$ Alexakis, S., Chen, D. Z., and Fournodvalos, G., "Singular Ricci solitons and their stability under the Ricci flow," Comm. Partial Diff. Eqs. 40(12), 2123-2172 (2015).

${ }^{2}$ Bernstein, J. and Mettler, T., "Two-dimensional gradient Ricci solitons revisited," Int. Math. Res. Not. IMRN 2015(1), 78-98.

${ }^{3}$ Betancourt de la Parra, A., "Painlevé analysis of the Bryant soliton," e-print arXiv:1310.7254 (math.DG).

${ }^{4}$ Buzano, M., Dancer, A. S., Gallaugher, M., and Wang, M., "A family of steady Ricci solitons and Ricci-flat metrics," Comm. Anal. Geom. 23(3), 611-638 (2015).

${ }^{5}$ Chow, B., Chu, S. C., Glickenstein, D., Guenther, C., Isenberg, J., Ivey, T., Knopf, D., Lu, P., Luo, F., and Ni, L., "The Ricci flow: Techniques and applications, I: Geometric aspects," in Mathematical Surveys and Monographs (American Mathematical Society, 2007), Vol. 135.

${ }^{6}$ Dancer, A., Hall, S., and Wang, M., "Cohomogeneity one shrinking Ricci solitons: An analytic and numerical study,” Asian J. Math. 17(1), 33-61 (2013).

${ }^{7}$ Dancer, A. and Wang, M., "The cohomogeneity one Einstein equations from the Hamiltonian viewpoint," J. Reine Angew. Math. 524, 97-128 (2000).

${ }^{8}$ Dancer, A. and Wang, M., "Superpotentials for the cohomogeneity one Einstein equations," Commun. Math. Phys. 260, 75-115 (2005).

${ }^{9}$ Dancer, A. and Wang, M., "Classification of superpotentials," Comm. Math. Phys. 284, 583-647 (2008)

${ }^{10}$ Dancer, A. and Wang, M., "New examples of non-Kähler Ricci solitons," Math. Res. Lett. 16, 349-363 (2009).

${ }^{11}$ Dancer, A. and Wang, M., "Non-Kähler expanding Ricci solitons," Int. Math. Res. Not. 2009(6), 1107-1133.

12 Dancer, A. and Wang, M., "On Ricci solitons of cohomogeneity one," Ann. Global Anal. Geom. 39, 259-292 (2011).

${ }^{13}$ Dancer, A. and Wang, M., "Classifying superpotentials: Three summands case," J. Geom. Phys. 61, 675-692 (2011).

${ }^{14}$ Goriely, A., "Integrability and nonintegrability of dynamical systems," in Advanced Series in Nonlinear Dynamics (World Scientific, 2001), Vol. 19

${ }^{15}$ Hamilton, R. S., "The formation of singularities in the Ricci flow," Surv. Differ. Geom. 2, 7-136 (1995).

${ }^{16}$ Ivey, T., "New examples of complete Ricci solitons," Proc. AMS 122, 241-245 (1994).

${ }^{17}$ Perelman, G., "The entropy formula for the Ricci flow and its geometric applications," e-print arXiv:math.DG/0211159.

${ }^{18}$ Prelle, M. J. and Singer, M. F., "Elementary first integrals of differential equations," Trans. Am. Math. Soc. 279, 215-229 (1983). 